\title{
Painful Psoriasiform Plaques
}

\author{
Maria Amoreth R. Gozo, MD; Iviensan F. Manalo, MD; Justin T. Cheeley, MD
}

\begin{abstract}
Eligible for 1 MOC SA Credit From the ABD
This Photo Challenge in our print edition is eligible for 1 self-assessment credit for Maintenance of Certification from the American Board of Dermatology (ABD). After completing this activity, diplomates can visit the ABD website (http://www.abderm.org) to self-report the credits under the activity title "Cutis Photo Challenge." You may report the credit after each activity is completed or after accumulating multiple credits.
\end{abstract}
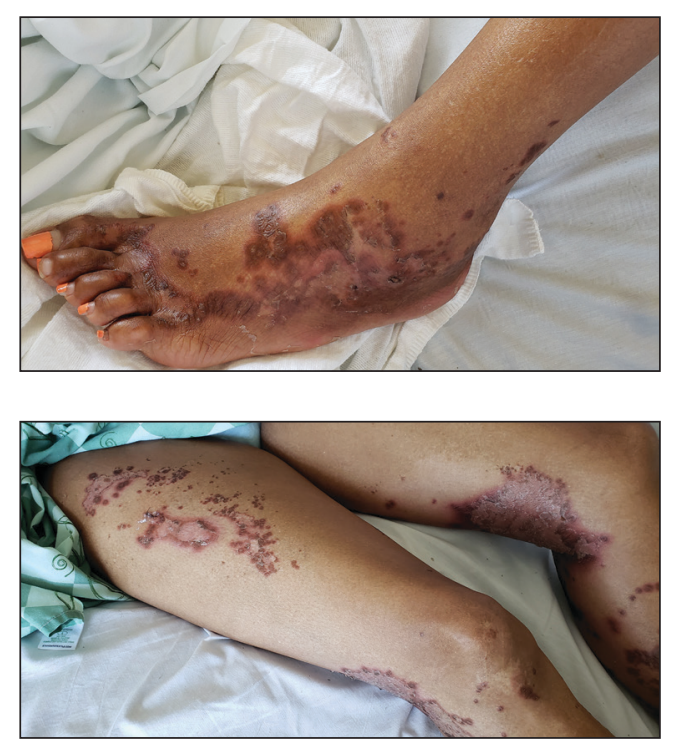

A 45-year-old woman presented to the emergency department with a painful skin eruption and malaise of 5 weeks' duration. She had an orthotopic liver transplant 5 years prior for endstage liver disease due to mixed nonalcoholic and alcoholic steatohepatitis and was on mycophenolate mofetil and tacrolimus for graft rejection prophylaxis. Her medical history also included Roux-en-Y gastric bypass 15 years prior, alcohol use disorder, hypothyroidism, and depression.

The exanthem began on the legs as pruritic, red, raised, exudative lesions that gradually crusted. Over the 2 weeks prior to the current presentation, the rash became tender as it spread to the feet, thighs, perianal skin, buttocks, and elbows. Triamcinolone ointment prescribed for a presumed nummular dermatitis effected marginal benefit. A review of systems was notable for a 15-pound weight loss over several weeks; lowgrade fever of 3 days' duration; epigastric abdominal pain; and long-standing, frequent defecation of oily, foul-smelling feces.

Physical examination revealed a combination of flat-topped, violaceous papules and serpiginous, polycyclic, annular plaques coalescing to form larger psoriasiform plaques with hyperkeratotic rims and dusky borders on the dorsal aspect of the feet (top), lateral ankles, legs (bottom), lateral thighs, buttocks, perianal skin, and elbows. Bilateral angular cheilitis, a smooth and fissured tongue, and pitting of all fingernails were noted.

\section{WHAT'S YOUR DIAGNOSIS? \\ a. acquired acrodermatitis enteropathica \\ b. Bazex syndrome (acrokeratosis paraneoplastica) \\ c. necrolytic acral erythema \\ d. necrolytic migratory erythema \\ e. psoriasis vulgaris}

PLEASE TURN TO PAGE 201 FOR THE DIAGNOSIS

Dr. Gozo is from the USS Anchorage, Naval Base San Diego, California. Drs. Manalo and Cheeley are from the Department of Dermatology, Emory University School of Medicine, Atlanta, Georgia. Dr. Cheeley also is from the Department of Medicine.

The authors report no conflict of interest.

The views expressed are those of the authors and do not reflect the opinions of the USS Anchorage (LPD-23), the US Navy, or the US Government. Correspondence: Maria Amoreth R. Gozo, MD, Health Services Department, USS Anchorage (LPD 23) FPO AP 96660 (amorethgozo@gmail.com). doi:10.12788/cutis.0356 


\section{THE DIAGNOSIS:}

\section{Acquired Acrodermatitis Enteropathica}

A punch biopsy of an elevated scaly border of the rash on the thigh revealed parakeratosis, absence of the granular layer, and epidermal pallor with psoriasiform and spongiotic dermatitis (Figure). Serum zinc levels were $60.1 \mu \mathrm{g} / \mathrm{dL}$ (reference range, $75.0-120.0 \mu \mathrm{g} / \mathrm{dL}$ ), suggestive of a nutritional deficiency dermatitis. Laboratory and histopathologic findings were most consistent with a diagnosis of acquired acrodermatitis enteropathica (AE).

Acrodermatitis enteropathica has been associated with Roux-en-Y gastric bypass and alcohol use disorder working synergistically to cause malabsorption and malnutrition, respectively. ${ }^{1}$ Zinc functions in the structural integrity, wound healing, and anti-inflammatory properties of the skin. There is a $17.3 \%$ risk for hypozincemia worldwide; in developed nations there is an estimated $3 \%$ to $10 \%$ occurrence rate. ${ }^{2}$ Acrodermatitis enteropathica can be classified as either acquired or hereditary. Both classically present as a triad of acral dermatitis, diarrhea, and alopecia, though the complete triad is seen in $20 \%$ of cases.

Hereditary AE is an autosomal-recessive disorder presenting in infancy that results in the loss of a zinc transporter. In contrast, acquired AE occurs later in life and usually is seen in patients who have decreased intake, malabsorption, or excessive loss of zinc. ${ }^{4}$ Acrodermatitis enteropathica is observed in individuals with conditions such as anorexia nervosa, pancreatic insufficiency, celiac disease, Crohn disease, or gastric bypass surgery (as in our case) and alcohol recidivism. In eanly disease, AE often presents with angular cheilitis and paronychia, but if left untreated, it can progress to mental status changes, hypogonadism, and depression. ${ }^{4}$

Acrodermatitis enteropathica presents as erythematous, erosive, scaly plaques or a papulosquamous psoriasiform rash with well-demarcated borders typically involving the orificial, acral, and intertriginous areas of the body. ${ }^{1,4}$ Acrodermatitis enteropathica belongs to a family of deficiency dermatoses that includes pellagra, necrolytic acral erythema (NAE), and necrolytic migratory erythema (NME) ${ }^{5}$ It is important to distinguish AE from NAE, as they can present similarly with well-defined and tender psoriasiform lesions peripherally. Histologically, NAE mimics AE with psoriasiform hyperplasia with parakeratosis. ${ }^{6}$ Necrolytic acral erythema characteristically is associated with active hepatitis C infection, which was absent in our patient. ${ }^{7}$

Similar to AE, NME affects the perineal and intertriginous surfaces. ${ }^{8}$ However, necrolytic migratory erythema has cutaneous manifestations in up to $70 \%$ of patients with glucagonoma syndrome, which classically presents as a triad of NME, weight loss, and diabetes mellitus. ${ }^{5}$ Laboratory studies show marked hyperglucagonemia, and imaging reveals enteropancreatic neoplasia.

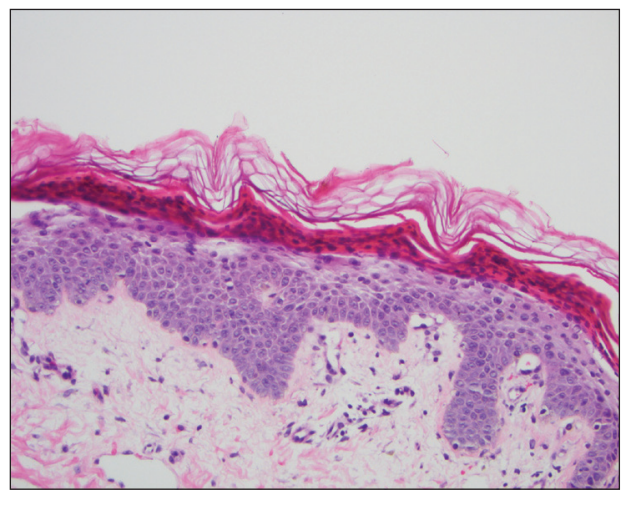

Acquired acrodermatitis enteropathica. Histopathology showed parakeratosis, absence of the granular layer, and epidermal pallor with psoriasiform and spongiotic dermatitis ( $H \& E$, original magnification $\times 20$ ).

Necrolytic migratory erythema will rapidly resolve once the glucagonoma has been surgically removed..$^{5}$

Bazex syndrome, or acrokeratosis paraneoplastica, is a paraneoplastic skin disease that is linked to underlying aerodigestive tract malignancies. Bazex syndrome clinically is characterized by hyperkeratotic and psoriasiform lesions favoring the ears, nails, and nose. ${ }^{9}$

Psoriasis vulgaris is a common chronic inflammatory skin condition that usually presents as well-demarcated plaques with silvery scale and observed pinpoint bleeding when layers of scale are removed (Auspitz sign). Lesions typically are found on the extensor surfaces of the body in addition to the neck, feet, hands, and trunk. Treatment of psoriasis vulgaris ranges from topical steroids for mild cases to systemic biologics for moderate to severe circumstances. ${ }^{10}$ In our patient, topical triamcinolone offered little relief.

Acrodermatitis enteropathica displays clinical and histologic characteristics analogous to many deficiency dermatoses and may represent a spectrum of disease. Because the clinicopathologic findings are nonspecific, it is critical to obtain a comprehensive history and maintain a high index of suspicion in patients with risk factors for malnutrition. The treatment for $\mathrm{AE}$ is supplemental oral zinc usually initiated at 0.5 to $1 \mathrm{mg} / \mathrm{kg}$ daily in children and 30 to $45 \mathrm{mg}$ daily in adults. ${ }^{3}$ Our patient initially was prescribed oral zinc supplementation; however, at 1-month follow-up, the rash had not improved. Failure of zinc monotherapy supports a multifactorial nutritional deficiency, which necessitated comprehensive nutritional appraisal and supplementation in our patient. Due to the steatorrhea, fecal pancreatic elastase levels were evaluated and were less than $15 \mu \mathrm{g} / \mathrm{g}$ (reference range, $\geq 201 \mu \mathrm{g} / \mathrm{g}$ ), confirming pancreatic exocrine insufficiency, a known complication of Roux-en-Y gastric bypass. ${ }^{11}$ Pancrelipase $500 \mathrm{U} / \mathrm{kg}$ per meal was added in addition to zinc oxide $40 \%$ paste to apply to the rash twice daily, with 
more frequent applications to the anogenital regions after bowel movements. The patient had substantial clinical improvement after 2 months.

\section{REFERENCES}

1. Shahsavari D, Ahmed Z, Karikkineth A, et al. Zinc-deficiency acrodermatitis in a patient with chronic alcoholism and gastric bypass: a case report. J Community Hosp Intern Med Perspect. 2014. doi:10.3402/jchimp.v4.24707

2. Kelly S, Stelzer JW, Esplin N, et al. Acquired acrodermatitis enteropathica: a case study. Cureus. 2017;9:E1667.

3. Guliani A, Bishnoi A. Acquired acrodermatitis enteropathica. JAMA Dermatol. 2019;155:1305.

4. Baruch D, Naga L, Driscoll M, et al. Acrodermatitis enteropathica from zinc-deficient total parenteral nutrition. Cutis. 2018;101:450-453.

5. van Beek AP, de Haas ER, van Vloten WA, et al. The glucagonoma syndrome and necrolytic migratory erythema: a clinical review. Eur J Endocrinol. 2004;151:531-537.
6. Botelho LF, Enokihara MM, Enokihara MY. Necrolytic acral erythema: a rare skin disease associated with hepatitis $C$ virus infection. An Bras Dermatol. 2016;91:649-651.

7. Abdallah MA, Ghozzi MY, Monib HA, et al. Necrolytic acral erythema: a cutaneous sign of hepatitis C virus infection. I Am Acad Dermatol. 2005;53:247-251.

8. Tolliver S, Graham J, Kaffenberger BH. A review of cutaneous manifestations within glucagonoma syndrome: necrolytic migratory erythema. Int J Dermatol. 2018;57:642-645.

9. Poligone B, Christensen SR, Lazova R, et al. Bazex syndrome (acrokeratosis paraneoplastica). Lancet. 2007;369:530.

10. Kupetsky EA, Keller M. Psoriasis vulgaris: an evidencebased guide for primary care. J Am Board Fam Med. 2013; 26:787-801.

11. Borbély Y, Plebani A, Kröll D, et al. Exocrine pancreatic insufficiency after Roux-en-Y gastric bypass. Surg Obes Relat Dis. 2016;12:790-794 